\title{
Open
}

see related editorial on page 1906

\section{Association Between the Location of Diverticular Disease and the Irritable Bowel Syndrome: A Multicenter Study in Japan}

\author{
Eiji Yamada, MD¹, Masahiko Inamori, MD¹, Eri Uchida, MD², Emiko Tanida, MD², Motoyoshi Izumi, MD², Kimiya Takeshita, MD³, \\ Tetsuro Fujii, MD4, Kazuto Komatsu, MD5, Jun Hamanaka, MD${ }^{6}$, Shin Maeda, MD¹, Akira Kanesaki, MD², Nobuyuki Matsuhashi, MD \\ and Atsushi Nakajima, MD ${ }^{1}$
}

OBJECTIVES: $\quad$ No previous reports have shown an association between location of diverticular disease (DD) and the irritable bowel syndrome (IBS).

METHODS: We included 1,009 consecutive patients undergoing total colonoscopy in seven centers in Japan from June 2013 to September 2013. IBS was diagnosed using Rome III criteria, and diverticulosis was diagnosed by colonoscopy with transparent soft-short-hood. Left-sided colon was defined as sigmoid colon, descending colon, and rectum. Right-sided colon was defined as cecum, ascending colon, and transverse colon. We divided the patients into IBS and non-IBS groups and compared characteristics.

RESULTS: $\quad$ Patient characteristics included mean age, 64.2 \pm 12.9 years and male:female ratio, 1.62:1. Rightsided DD was identified in $21.6 \%$ of subjects. Left-sided and bilateral DD was identified in 6.6 and $12.0 \%$ of subjects, respectively. IBS was observed in $7.5 \%$ of subjects. Multiple logistic regression analysis showed left-sided DD (odds ratio, $3.1 ; 95 \%$ confidence interval $(\mathrm{CI}): 1.4-7.1 ; P=0.0060$ ) and bilateral DD (odds ratio, 2.6; $95 \% \mathrm{Cl}, 1.3-5.2 ; P=0.0070$ ) were independent risk factors for IBS. Right-sided DD was not a risk factor for IBS.

CONCLUSIONS: Our data showed that the presence of left-sided and bilateral DD, but not right-sided disease, was associated with a higher risk of IBS, indicating that differences in pathological factors caused by the location of the DD are important in the development of IBS. Clarifying the specific changes associated with left-sided DD could provide a better understanding of the pathogenic mechanisms of IBS (Trial registration \# R000012739).

Am J Gastroenterol 2014; 109:1900-1905; doi:10.1038/ajg.2014.323; published online 21 October 2014

\section{INTRODUCTION}

The irritable bowel syndrome (IBS) is a functional gastrointestinal disorder characterized by abdominal pain or discomfort associated with stool quality or frequency (1). The financial impact of IBS on society is high because of medical costs, absenteeism from work, and impaired health-related quality of life (2). In recent years, clinicians have observed an increasing overlap of clinical and pathophysiologic features that typically distinguish functional from organic disorders-that is, a blurring of the boundaries of the dualistic biomedical dichotomy (3). For major example, many patients with inflammatory bowel disease (IBD) in clinical remission continue to have symptoms of pain and diarrhea despite minimal or no ongoing inflammation. These patients may be considered to have overlap of IBD and IBS (4).

A subset of patients with colonic diverticular disease (DD) has chronic gastrointestinal symptoms, and some have a clinical diagnosis of IBS, but whether IBS and DD are linked is uncertain. Jung et al. (5) showed that the presence of colonic DD has been associated with higher risks of IBS and diarrhea-predominant IBS (D-IBS), whereas most patients with diverticulosis are asymptomatic. Cohen et al. (6) also reported that patients with a history of diverticulitis could be at risk for later development of IBS and

'Gastroenterology Division, Yokohama City University School of Medicine, Yokohama, Japan; ²Department of Gastroenterology, Machida Municipal Hospital, Machida, Tokyo, Japan; ${ }^{3}$ Department of Gastroenterology, International University of Health and Welfare Atami Hospital, Atami, Shizuoka, Japan; ${ }^{4}$ Department of Gastroenterology, Tokyo Metropolitan, Hiroo General Hospital, Shibuya-ku, Tokyo, Japan; ${ }^{5}$ Department of Gastroenterology, Yokosuka City Hospital, Yokosuka, Kanagawa, Japan; ${ }^{6}$ Department of Gastroenterology, Yokohama Minami Kyosai Hospital, Yokosuka, Kanagawa, Japan; ${ }^{7}$ Department of Gastroenterology, NTT Medical Center Tokyo, Gotanda, Tokyo, Japan. Correspondence: Atsushi Nakajima, MD, Gastroenterology Division, Yokohama City University School of Medicine, 3-9 Fuku-ura, Kanazawa-ku, Yokohama 236-0004, Japan. E-mail: nakajima-tky@umin.ac.jp

Received 12 February 2014; accepted 5 August 2014 
functional bowel disorders. These studies showed important epidemiological associations between DD and IBS. However, there are several points in developing a deeper understanding of the association between DD and IBS. Above all, previous studies were conducted in Western countries $(5,6)$. One major difference between colonic DD in the West and Asia is the predominant location. In European and US populations, DD arises mainly in the distal colon, with $90 \%$ of patients having sigmoid colon involvement and only approximately $15 \%$ having right-sided disease (7). However, in Asian populations, DD is mainly right-sided (8). Why is the location of DD important? Faucheron et al. (9) reported that rightsided localization of DD is associated with a risk of bleeding, Law et al. (10) showed that left-sided diverticulitis tended to become severe, and we showed that development of left-sided diverticulitis was associated with visceral obesity (11). These studies show that the risk of onset or severity of DD depends on the location. However, there are no previous reports showing an association between the location of DD and IBS. Also, gastrointestinal physiology may vary between Asian and Western populations because of differences in diet, sociocultural background, and genetic factors (12). Therefore, we must evaluate the association between DD and IBS in the Japanese population to develop a deeper understanding of the association between DD and IBS.

\section{METHODS}

This study was a multicenter study conducted with the approval of the Ethics Committee of Yokohama City University (approval number B130307045) and each center. Examination was performed after registering the trial with the University Hospital Medical Information Network in Japan (registration number R000012739).

\section{Subjects and survey method}

This study was performed in seven centers (Yokohama City University Hospital, Machida Municipal Hospital, Yokosuka City Hospital, Yokohama Minami Kyosai Hospital, NTT Medical Center Tokyo, International University of Health and Welfare Atami Hospital, and Tokyo Metropolitan Hiroo General Hospital) from June 2013 to September 2013. Each hospital is a flagship hospital in each region. Study subjects were consecutive Japanese patients who underwent total colonoscopy at each center, and the reason for colonoscopy was colon and rectal cancer screening. We excluded patients with active intra-abdominal and pelvic malignancy, ulcerative colitis, Crohn's disease, microscopic colitis, and other organic gastrointestinal disorders as in previous reports (5). We also excluded patients with a history of intestinal resection. The diagnosis of diverticulosis was established by colonoscopy with transparent soft-short-hood and diverticulosis was defined by the presence of one diverticulum or multiple diverticula. DD is the combination of a past history of diverticulitis and the diverticulosis. The location of DD was defined as follows: left-sided colon was defined as sigmoid colon, descending colon, and rectum, and right-sided colon was defined as cecum, ascending colon, and transverse colon.

\section{Diagnosis of IBS}

We used a questionnaire to diagnose IBS and to collect the necessary information before colonoscopy in all subjects. IBS: Subjects were classified as having IBS on the basis of the symptoms recorded in the questionnaire. IBS and the IBS subtype were defined using Rome III criteria (1). The definition applied required recurrent abdominal pain or discomfort frequency of at least 2 days a week with at least two of the following (1): improvement with defecation (2); onset associated with a change in frequency of stool; and (3) onset associated with a change in the form of stool. Stool form was assessed using the Bristol scale (13). IBS was sub-typed as (i) D-IBS: IBS with loose or watery stools for at least $25 \%$ of bowel movements, and hard or lumpy stool for $<25 \%$ of the bowel movements; (ii) constipation-predominant IBS: IBS with hard or lumpy stool for at least $25 \%$ of the bowel movements, and loose or watery stools for $<25 \%$ of the bowel movements; (iii) mixed IBS: meeting the criteria for both D-IBS and constipation-predominant IBS; and (iv) un-subtype IBS (U-IBS): IBS not meeting the criteria for the other three categories (1).

\section{Additional information}

Additional information was gathered from the questionnaire before undergoing colonoscopy:

(i) Demographics: age and gender.

(ii) Body mass index (BMI): based on self-recording of height and current weight.

(iii) Mental health status was evaluated using SF-8 (14). The SF-8 is a generic questionnaire derived from the longer 36-item Short-Form Health Survey (SF-36) and is developed to estimate health-related quality of life (HRQOL) based on scores from eight domains and two summaries. Results obtained from the SF-8 show a high correlation with the SF-36. Scores for the physical and mental component summaries (MCS) are calculated according to the manual for the Japanese version of the SF-8. A score of 50 is the mean for the Japanese general population across the eight domains and two scores; lower scores indicate a poorer HRQOL. Therefore, we defined that an MCS $<50$ defined poor mental status.

(iv) Laxative use (yes or no).

(v) Socioeconomic factors: marital status (married or not).

(vi) Previous history of cholecystectomy (yes or no).

(vii) Use of cigarettes and alcohol: current cigarette habit (yes/no) and alcohol (yes for current drinking habit, no for never or no current use).

(viii) Medication history.

\section{Statistical analysis}

Statistical analysis was performed using Excel-Toukei 2010 software for Windows (Social Survey Research Information, Tokyo, Japan). Differences between the measured and group values were analyzed using the $\chi^{2}$-test and Student's $t$-test. A multivariable analysis was performed using a conditional logistic regression model, and $P$ values $<0.05$ were considered statistically significant. 


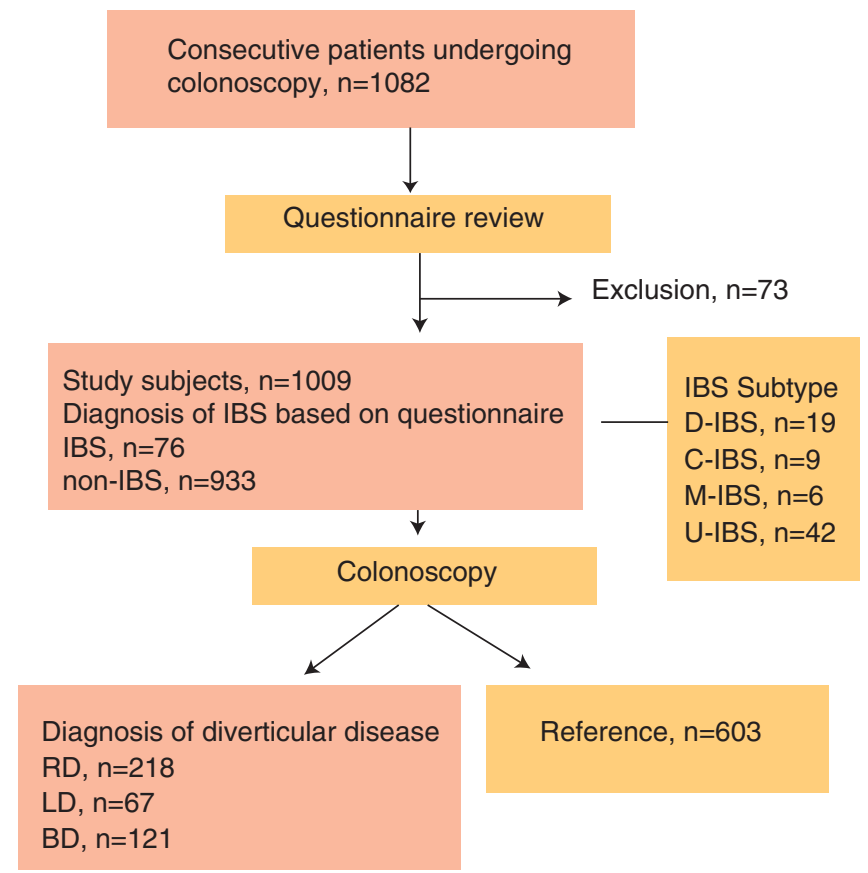

Figure 1. The flow diagram for this study. BD, bilateral diverticular disease; IBS, irritable bowel syndrome; LD, left-sided diverticular disease; $n$, number.

\section{RESULTS}

\section{Location of DD and IBS}

Figure 1 shows the flow diagram of this study. We initially enrolled 1,082 subjects and 73 subjects met the exclusion criteria. A final total of 1,009 subjects were enrolled in this study, and Table 1 shows the patient characteristics. The male-to-female ratio was $1.62: 1$, with a mean age of $64.2 \pm 12.9$ years (range, $20-85$ years). Table 2 shows a comparison of the IBS group $(n=76)$ and the nonIBS group $(n=933)$. The prevalence of laxative use $(P=0.0010)$, MCS $<50(P=0.0001), \mathrm{LD}(P=0.0002)$, and BD $(P=0.0307)$ had $P$ values of $<0.05$. We then performed a multiple logistic regression analysis using parameters with a $P$ value of $<0.05$ identified by univariate regression analysis in addition to the following parameters: G65 years of age, male, RD, and NSAID use $(P=0.0654)$. Table 3 shows the results of the multiple logistic regression analysis. Multiple logistic regression analysis showed MCS (<50) (odds ratio: $3.7,95 \%$ confidence interval (CI): 2.0-6.9, $P=0.0001)$, LD (odds ratio: $3.1,95 \% \mathrm{CI}: 1.4-7.1, P=0.0060$ ), and $\mathrm{BD}$ (odds ratio: 2.6, 95\% CI: $1.3-5.2 ; P=0.0070$ ) were independent risk factors for IBS. However, RD was not an independent risk factor for IBS (odds ratio: 0.9 , 95\% CI: $0.5-1.9, P=0.8873$ ).

\section{Location of DD and IBS subtype}

We also studied the association between IBS subtype and DD location. We calculated the odds ratio for $\mathrm{RD}, \mathrm{LD}$, and $\mathrm{BD}$, with relative risk of IBS subtype compared with no IBS group. Table 4 shows the association between IBS subtype and location of DD. Left-sided DD was an independent risk factor for D-IBS and

\section{Table 1. Patients' characteristics}

\begin{tabular}{lc} 
& $n=1,009$ \\
\hline Age (s.d.; years) & $64.2 \pm 12.9$ \\
Gender (M:F) & $1.62: 1$ \\
Mean BMI (s.d.) & $22.9 \pm 3.5$ \\
Smoker \% & 17.4 \\
Alcohol \% & 41.8 \\
Laxative use \% & 14.8 \\
NSAIDs \% & 1.5 \\
Aspirin \% & 7.0 \\
Previous cholecystectomy \% & 5.0 \\
History of acute colonic diverticulitis & $5.2 \%$ \\
Married \% & 79.6 \\
MCS (<50) \% & 51.4 \\
RD & $21.6 \%$ \\
LD & $6.6 \%$ \\
BD & $12.0 \%$ \\
IBS & $7.5 \%$ \\
\hline BD, bilateral diverticular disease; BMI, body mass index; IBS, irritable bowel \\
syndrome; LD, left-sided diverticular disease; MCS, mental component sum- \\
mary; $n$, number; NSAID, non-steroidal anti-inflammatory drug; RD, right-sided \\
diverticular disease. & \\
\hline
\end{tabular}

U-IBS (odds ratio: 8.8 , 95\% CI: 2.5 -30.8; odds ratio: $3.1,95 \%$ CI: $1.2-8.2$, respectively).

\section{DISCUSSION}

RD was identified in $21.6 \%$ of the subjects in our study, and LD and BD were identified in 6.6 and $12.0 \%$ of the subjects, respectively. IBS was diagnosed in $7.5 \%$ of the subjects (Figure 2), and a history of acute colonic diverticulitis was present in $5.2 \%$ of the subjects. We showed that LD and BD were independent risk factors for IBS, and we also showed that LD was associated with D-IBS and U-IBS. Our most interesting finding was that RD was not associated with IBS. It is difficult to explain why we found no relation between RD and IBS. However, we propose some hypotheses.

A difference in visceral hypersensitivity induced by DD is one hypothesis. Visceral hypersensitivity is also an important factor for development of IBS (15). Patients with symptomatic uncomplicated DD were shown to have visceral hypersensitivity (16). Visceral hypersensitivity has been reported to be caused by lowgrade inflammation and neuropeptide changes $(17,18)$. The lack of visceral hypersensitivity in patients with $\mathrm{RD}$ may be due to differences between the dominant nerves of the colon on the proximal and distal sides. In the colon, afferent nerve fibers reach the central nervous system via parasympathetic pathways and spinal afferent nerves, both of which display mechano- and chemo-sensitivity (19). 


\begin{tabular}{|c|c|c|c|}
\hline & IBS ( $n=76)$ & $\begin{array}{l}\text { non-IBS } \\
(n=933)\end{array}$ & $P$ value \\
\hline Age (s.d.; years) & $65.8 \pm 13.7$ & $64.1 \pm 12.8$ & $0.2649^{s}$ \\
\hline$\leq 65$ years & $64.5 \%$ & $56.6 \%$ & $0.1818^{c}$ \\
\hline Gender (M:F) & $1.17: 1$ & $1.67: 1$ & $0.1406^{c}$ \\
\hline Mean BMI (s.d.) & $22.4 \pm 3.4$ & $23.0 \pm 3.5$ & $0.1822^{\mathrm{s}}$ \\
\hline Smoker \% & 14.5 & 17.8 & $0.4645^{c}$ \\
\hline Alcohol \% & 42.1 & 41.8 & $0.9587^{c}$ \\
\hline Laxative use \% & 27.6 & 13.7 & $0.0010^{\mathrm{c} *}$ \\
\hline NSAIDs \% & 3.9 & 1.3 & $0.0653^{c}$ \\
\hline Aspirin \% & 7.7 & 7.0 & $0.7610^{c}$ \\
\hline Previous cholecystectomy \% & 3.9 & 5.0 & $0.6737^{c}$ \\
\hline $\begin{array}{l}\text { History of acute colonic } \\
\text { diverticulitis }\end{array}$ & $2.6 \%$ & $5.4 \%$ & $0.3011^{c}$ \\
\hline Married \% & 77.6 & 80.9 & $0.4848^{c}$ \\
\hline $\operatorname{MCS}(<50) \%$ & 79.1 & 49.0 & $0.0001^{c *}$ \\
\hline RD & $18.4 \%$ & $21.9 \%$ & $0.4830^{c}$ \\
\hline LD & $18.4 \%$ & $5.68 \%$ & $0.0002^{\mathrm{C} *}$ \\
\hline $\mathrm{BD}$ & $19.7 \%$ & $11.4 \%$ & $0.0307^{* *}$ \\
\hline \multicolumn{4}{|c|}{$\begin{array}{l}\text { BD, bilateral diverticular disease; BMI, body mass index; IBS, irritable bowel syn- } \\
\text { drome; LD, left-sided diverticular disease; MCS, mental component summary; } n \text {, } \\
\text { number; NSAID, non-steroidal anti-inflammatory drug; RD, right-sided diverticular } \\
\text { disease. } \\
{ }^{c} \chi^{2} \text {-Test. } \\
{ }^{s} \text { Student's } t \text { test. } \\
{ }^{*}(P<0.05) \text {. }\end{array}$} \\
\hline
\end{tabular}

The parasympathetic fibers convey sensory information from the proximal colon (cecum, ascending colon, and 2/3 of the transverse colon) via the vagus nerve to cell bodies in the nodose and jugular ganglia and from the distal colon (1/3 of the transverse colon, sigmoid colon, descending colon, and rectum) via the pelvic splanchnic nerves (19-21). Enteric nerve changes caused by low-grade inflammation may vary according to the dominant nerve, which may explain the lack of relationship between IBS and RD. However, further investigations are necessary to prove this hypothesis.

When we studied the association between IBS subtype and location of DD, we showed that U-IBS and D-IBS were significantly associated with LD. Previous studies have also shown that D-IBS was associated with DD (5). Our data showed that U-IBS was also significantly associated with LD, indicating the possibility that the impact of LD on IBS is related not only to bowel habits but also to other factors such as pain or discomfort.

Previous reports showed that psychosocial factors are important in the development of IBS (22); therefore, we used MCS to evaluate mental health. Previously, Kaji et al. (23) reported a positive association between low MCS and IBS patients overlapping Gastroesophageal reflux disease, functional dyspepsia, and our data showed that low MCS was an important factor in the development of IBS.
Table 3. Results of the multiple logistic regression analysis for independent risk for IBS

$\begin{array}{lccc} & \text { Odds ratio } & 95 \% \mathbf{C l} & \boldsymbol{P} \text { value } \\ \text { Male } & 0.7 & 0.4-1.1 & 0.1466 \\ \leq 65 \text { years } & 1.2 & 0.7-2.0 & 0.5863 \\ \text { NSAIDs } & 2.0 & 0.5-8.1 & 0.3069 \\ \text { MCS }(<50) & 3.7 & 2.0-6.9 & 0.0001^{*} \\ \text { RD } & 0.9 & 0.5-1.9 & 0.8873 \\ \text { LD } & 3.1 & 1.4-7.1 & 0.0060^{*} \\ \text { BD } & 2.6 & 1.3-5.2 & 0.0070^{*}\end{array}$

$\mathrm{Cl}$, confidence interval; NSAID, non-steroidal anti-inflammatory drug; MCS, mental component summary; RD, right-sided diverticular disease; LD, left-sided diverticular disease; BD, bilateral diverticular disease.

${ }^{*}(P<0.05)$.

Our study has several limitations. First, our study target comprised subjects who underwent colonoscopy for colon and rectal cancer screening. Therefore, our study subjects may be more symptomatic compared with general populations. Second, it is said that the detection rate of diverticulum by colonoscopy was inferior compared with barium enema (24). Therefore, for the purposes of accuracy, we used a transparent soft-short-hood on the scope for colonoscopy. Previous reports have shown that a transparent soft-short-hood allows detection of small lesions (25). Third, the number of subjects with IBS was too small to draw conclusions about the association between IBS subtype and DD location. To address this limitation, we have been enrolling a larger number of study subjects to increase the accuracy. Fourth, the ratio of IBS subtype to gender in our study differed from previous reports. In Japan, D-IBS was common in males and constipation-predominant IBS was common in females in younger age groups (26). However, our data showed that U-IBS was most common. This is likely because the average age of our subjects was higher than that of the peak age at onset. Fifth, the classification of IBS based on patient recall is highly inaccurate. It may have been better to use a 7-day stool diary, allowing investigators to classify each subject's bowel habits (27). Sixth, we found that the proportion of males was higher compared with females in our study cohort. Probably, this is because of social background in Japan, in that more asymptomatic males than females undergo a total colonoscopy for colorectal cancer screening (28).

In summary, our data showed that the presence of $\mathrm{LD}$ and $\mathrm{BD}$ was both associated with a higher risk of IBS. However, RD showed no relationship with IBS. Therefore, differences in pathological factors caused by the location of the DD are important in the development of IBS. Clarifying the specific changes associated with left-sided DD could provide a better understanding of the pathogenic mechanisms of IBS. 
Table 4. Association between IBS subtype and location of DD

\begin{tabular}{|c|c|c|c|c|c|c|}
\hline & $\mathrm{RD}(n=218)$ & & LD $(n=67)$ & & $\mathrm{BD}(n=121)$ & \\
\hline \multirow[t]{2}{*}{ D-IBS (\%) } & 1.4 & & 9.0 & & 2.5 & \\
\hline & $1.3(0.3-5.1)$ & 0.7041 & $8.8(2.5-30.8)$ & $0.0006^{*}$ & $2.8(0.7-11.3)$ & 0.1524 \\
\hline \multirow[t]{2}{*}{ C-IBS (\%) } & 0.9 & & 1.5 & & 1.7 & \\
\hline & $1.5(0.3-8.1)$ & 0.653 & $2.3(0.3-21.1)$ & 0.4621 & $2.7(0.5-14.9)$ & 0.2574 \\
\hline & $5.5(0.5-60.8)$ & 0.1674 & $8.2(0.5-138.7)$ & 0.1466 & $9.2(0.8-104.3)$ & 0.0726 \\
\hline \multirow[t]{2}{*}{ U-IBS (\%) } & 3.2 & & 9.0 & & 6.6 & \\
\hline & $0.9(0.4-2.2)$ & 0.8806 & $3.1(1.2-8.2)$ & $0.0235^{*}$ & $2.1(0.9-4.8)$ & 0.1021 \\
\hline
\end{tabular}

BD, bilateral diverticular disease; Cl, confidence interval; C-IBS, constipation-predominant IBS; DD, diverticular disease; IBS, irritable bowel syndrome; LD, left-sided diverticular disease; M-IBS, mixed IBS; $n$, number; OR, odds ratio; RD, right-sided diverticular disease; U-IBS, un-subtype IBS.

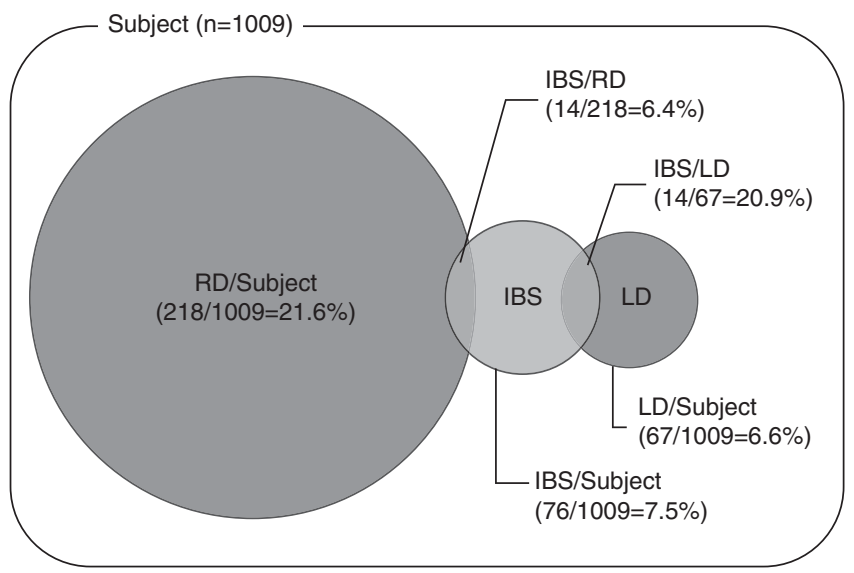

Figure 2. Overlaps of RD and LD with IBS. IBS, irritable bowel syndrome; $n$, number; LD, left-sided diverticular disease; RD, right-sided diverticular disease.

\section{ACKNOWLEDGMENTS}

We thank Masataka Tagri for his help with the statistical analysis. The authors also thank the nurses and staff in each hospital for their help in collecting data.

\section{CONFLICT OF INTEREST}

Guarantor of the article: Atsushi Nakajima, MD.

Specific author contributions: Eiji Yamada drafted the article. Eri Uchida, Emiko Tanida, Motoyoshi Izumi, Kimiya Takeshita, Tetsuro Fujii, Kazuto Komatsu, and Jun Hamanaka collected and analyzed the data. Akira Kanesaki critically revised the article for important intellectual content and analyzed and interpreted the data. Masahiko Inamori analyzed and interpreted the data and critically revised the article for important intellectual content. Shin Maeda, Nobuyuki Matsuhashi, and Atsushi Nakajima conceived and designed the study and gave final approval for the article. All authors have approved the final version of the article, including the authorship list.

Financial support: None.

Potential competing interests: None.

\section{Study Highlights}

\section{WHAT IS CURRENT KNOWLEDGE}

Both colonic diverticular disease (DD) and the irritable bowel syndrome (IBS) are common in the population.

The risk of onset or severity of DD depends on the location. No previous reports have shown an association between location of DD and the IBS.

\section{WHAT IS NEW HERE}

The presence of Left-sided DD and Bilateral-sided DD was both associated with a higher risk of IBS.

Right-sided DD showed no relationship with IBS.

Left-sided DD was associated with diarrhea-predominant IBS and un-subtype IBS.

\section{REFERENCES}

1. Longstreth GF, Thompson WG, Chey WD et al. Functional showebowel disorders. Gastroenterology 2006;130:1480-91.

2. Sandler RS, Everhart JE, Donowitz $\mathrm{M}$ et al. The burden of selected digestive diseases in the United States. Gastroenterology 2002;122:1500-11.

3. Drossman DA. Functional GI disorders: what's in a name? Gastroenterology 2005;128:1771-2.

4. Halpin SJ, Ford AC. Prevalence of symptoms meeting criteria for irritable bowel syndrome in inflammatory bowel disease: systematic review and meta-analysis. Am J Gastroenterol 2012;107:1474-82.

5. Jung HK, Choung RS, Locke GR 3rd et al. Diarrhea-predominant irritable bowel syndrome is associated with diverticular disease: a population-based study. Am J Gastroenterol 2010;105:652-61.

6. Cohen E, Fuller G, Bolus R et al. Increased risk for irritable bowel syndrome after acute diverticulitis. Clin Gastroenterol Hepatol 2013;11:1614-9.

7. Parks TG. Natural history of diverticular disease of the colon. Clin Gastroenterol 1975;4:53-69.

8. Miura S, Kodaria S, Aoki $\mathrm{H}$ et al. Bilateral type diverticular disease of the colon. Int J Colorect Dis 1996;11:71-5.

9. Faucheron JL, Roblin X, Bichard P et al. The prevalence of right-sided colonic diverticulosis and diverticular haemorrhage. Colorectal Dis 2013;15:e266-e270.

10. Law WL, Lo CY, Chu KW. Emergency surgery for colonic diverticulitis: differences between right-sided and left-sided lesions. Int J Colorectal Dis 2001;16:280-4. 
11. Yamada E, Ohkubo H, Higurashi T et al. Visceral obesity as a risk factor for left-sided diverticulitis in Japan: a multicenter retrospective study. Gut Liver 2013;7:532-8.

12. Lee OY. Asian motility studies in irritable bowel syndrome. J Neurogastroenterol Motil 2010;16:120-30.

13. Riegler G, Esposito I. Bristol scale stool form. A still valid help in medical practice and clinical research. Tech Coloproctol 2001;5:163-4.

14. Fukuhara S, Suzukamo Y. Manual of the SF-8 Japanese edition Institute for Health Outcomes and Process Evaluation Research: Kyoto, 2004.

15. Mertz H, Naliboff B, Munakata J et al. Altered rectal perception is a biological marker of patients with irritable bowel syndrome. Gastroenterology 1995;109:40-52.

16. Clemens CH, Samsom M, Roelofs J et al. Colorectal visceral perception in diverticular disease. Gut 2004;53:717-22.

17. Simpson J, Sundler F, Humes DJ et al. Post inflammatory damage to the enteric nervous system in diverticular disease and its relationship to symptoms. Neurogastroenterol Motil 2009;21:847-e58.

18. Humes DJ, Simpson J, Smith J et al. Visceral hypersensitivity in symptomatic diverticular disease and the role of neuropeptides and low grade inflammation. Neurogastroenterol Motil 2012;24:318-e163.

19. Blackshaw LA, Brookes SJ, Grundy D et al. Sensory transmission in the gastrointestinal tract. Neurogastroenterol Motil 2007;19(1 Suppl):1-19.

20. Camilleri M, Ford MJ. Review article: colonic sensorimotor physiology in health, and its alteration in constipation and diarrhoeal disorders. Aliment Pharmacol Ther 1998;12:287-302.

21. O'Brien MD, Phillips SF. Colonic motility in health and disease. Gastroenterol Clin North Am 1996;25:147-62.
22. Drossman DA, McKee DC, Sandler RS et al. Psychosocial factors in the irritable bowel syndrome. A multivariate study of patients and nonpatients with irritable bowel syndrome. Gastroenterology 1988;95:701-8.

23. Kaji M, Fujiwara Y, Shiba M et al. Prevalence of overlaps between GERD, FD and IBS and impact on health-related quality of life. J Gastroenterol Hepatol 2010;25:1151-6.

24. Niikura R, Nagata N, Shimbo T et al. Colonoscopy can miss diverticula of the left colon identified by barium enema. World J Gastroenterol 2013;19:2362-7.

25. Kondo S, Yamaji Y, Watabe $\mathrm{H}$ et al. A randomized controlled trial evaluating the usefulness of a transparent hood attached to the tip of the colonoscope. Am J Gastroenterol 2007;102:75-81.

26. Miwa H. Prevalence of irritable bowel syndrome in Japan: internet survey using Rome III criteria. Patient Prefer Adherence 2008;2:143-7.

27. Palsson OS, Baggish JS, Turner MJ et al. IBS patients show frequent fluctuations between loose/watery and hard/lumpy stools: implications for treatment. Am J Gastroenterol 2012;107:286-95.

28. Morikawa T, Kato J, Yamaji Y et al. A comparison of the immunochemical fecal occult blood test and total colonoscopy in the asymptomatic population. Gastroenterology 2005;129:422-8.

(1) (2) (2) This work is licensed under a Creative Commons Attricc. cense. To view a copy of this license, visit http://creativecommons. org/licenses/by-nc-sa/3.0/ 